\title{
Comites Villageois Et Problématique De La Gestion Des Points D'eau Dans L'arrondissement De Bayangam (Ouest-Cameroun)
}

\author{
Hervé Tchekote \\ Chargé de Cours au Département de Géographie-Aménagement- \\ Environnement de l'Université de Dschang-Cameroun \\ Angeline Noufin Poka \\ Chercheure au Laboratoire de Climatologie et de Recherches \\ Environnementales, Département de Géographie-Aménagement- \\ Environnement, Université de Dschang-Cameroun

\section{Fridolin Brice Nicolas Atekoa Mbarga} \\ Chercheur au Laboratoire de Climatologie et de Recherches \\ Environnementales, Département de Géographie-Aménagement- \\ Environnement, Université de Dschang-Cameroun
}

\begin{abstract}
Households in Bayangam district in the west high land of Cameroon face many difficulties, one of the most important of which is access to water resources in terms of quality and quantity. Most people travel long distances in search of water, which is sometimes not reassuring. However, many boreholes as well as Village Committees of Water Point Management (CVGPE) have been set up in this locality. From this observation, the hypothesis according to which the existence of the Village Management Committees does not ensure the sustainable management of the water points in Bayangam was formulated. To verify this hypothesis, literature searches as well as direct observations in the study area were first conducted. Then, survey questionnaires from 150 households were administered. Finally, interviews with 14 resource persons representing the key players were conducted. The results of the study show that many CVGPEs have been established in the Bayangam district in recent decades, with the aim of ensuring better management of the water points set up by a multitude of stakeholders. However, most of these water points are almost non-functional or poorly maintained. Faced with this situation, it is important: to carry out a complete diagnosis of the works for a rehabilitation, to improve the choice of the members for more efficient services, to encourage a thorough involvement of
\end{abstract}


the beneficiaries and a more remarkable support of the Bayangam commune to manage water points for a sustainable supply of populations

Keywords : Bayangam, Village Management Committees, Water Point Management.

\section{Résumé}

Les ménages de l'arrondissement de Bayangam sur les hautes terres de l'Ouest Cameroun font face à de nombreuses difficultés dont l'une des plus importantes est l'accès à la ressource en eau en termes de qualité et de quantité. Les populations pour la majorité parcourent de longues distances à la recherche de l'eau qui parfois n'est pas de qualité rassurante pourtant, de nombreux forages ainsi que des Comités Villageois de Gestion des Point d'Eau (CVGPE) ont été mis en place dans cette localité. De ce constat, l'hypothèse selon laquelle l'existence des Comités Villageois de Gestion n'assure pas de manière pérenne et durable la gestion des points d'eau à Bayangam a été formulée. Pour vérifier cette hypothèse, des recherches documentaires ainsi que des observations directes dans la zone d'étude ont d'abord été menées. Ensuite, des questionnaires d'enquêtes auprès de 150 ménages ont été administrés. Enfin, des entretiens auprès de 14 personnes ressources représentant les acteurs clés ont été menés. Les résultats de l'étude montrent que de nombreux CVGPE ont été crées dans l'arrondissement de Bayangam au cours de ces derniers décennies, avec comme objectif d'assurer une meilleure gestion des points d'eau mis en place par une multitude d'acteurs. Cependant, la plupart de ces points d'eau, sont pour près de la moitié non fonctionnels ou mal entretenus. Face à cette situation, il est important : de réaliser un diagnostic complet des ouvrages pour une réhabilitation, d'améliorer le choix des membres pour des services plus efficaces, d'encourager une implication approfondie des bénéficiaires et d'un soutien davantage remarquable de la commune de Bayangam à la gestion des points d'eau pour un approvisionnement pérenne des populations.

Mots clés : Bayangam, Comités Villageois de Gestion, Gestion des Points d'Eau.

\section{Introduction}

L'intérêt des sciences sociales pour les problématiques liées à l'eau (l'accès à l'eau potable, la diversité des modes de gouvernance, la gestion de la ressource) est récent. En effet, depuis les années 1990, les recherches dans ce domaine se foisonnent, se construisent et se consolident de façon continue (Baron et Tidjani, 2011). Si l'analyse des modes de gestion de l'eau constitue inéluctablement un champ d'investigation important (Schneier-Madanes, 
2011), surtout à l'heure où les changements climatiques pèsent sur les dynamiques socioéconomiques et politiques à travers le monde, il demeure vrai que la question de son accessibilité est un enjeu majeur de développement tant en milieu urbain que rural. Dans ces milieux, les populations se heurtent au quotidien à des problèmes d'hydrauliques.

Dans la perspective d'une gestion durable de l'eau potable dans certaines localités rurales, les acteurs notamment ceux de l'arrondissement de Bayangam $^{1}$ dans l'Ouest Cameroun (figure 1) s'organisent en comité de gestion, avec comme objectif de veiller à l'entretien des ouvrages hydrauliques qui les desservent.

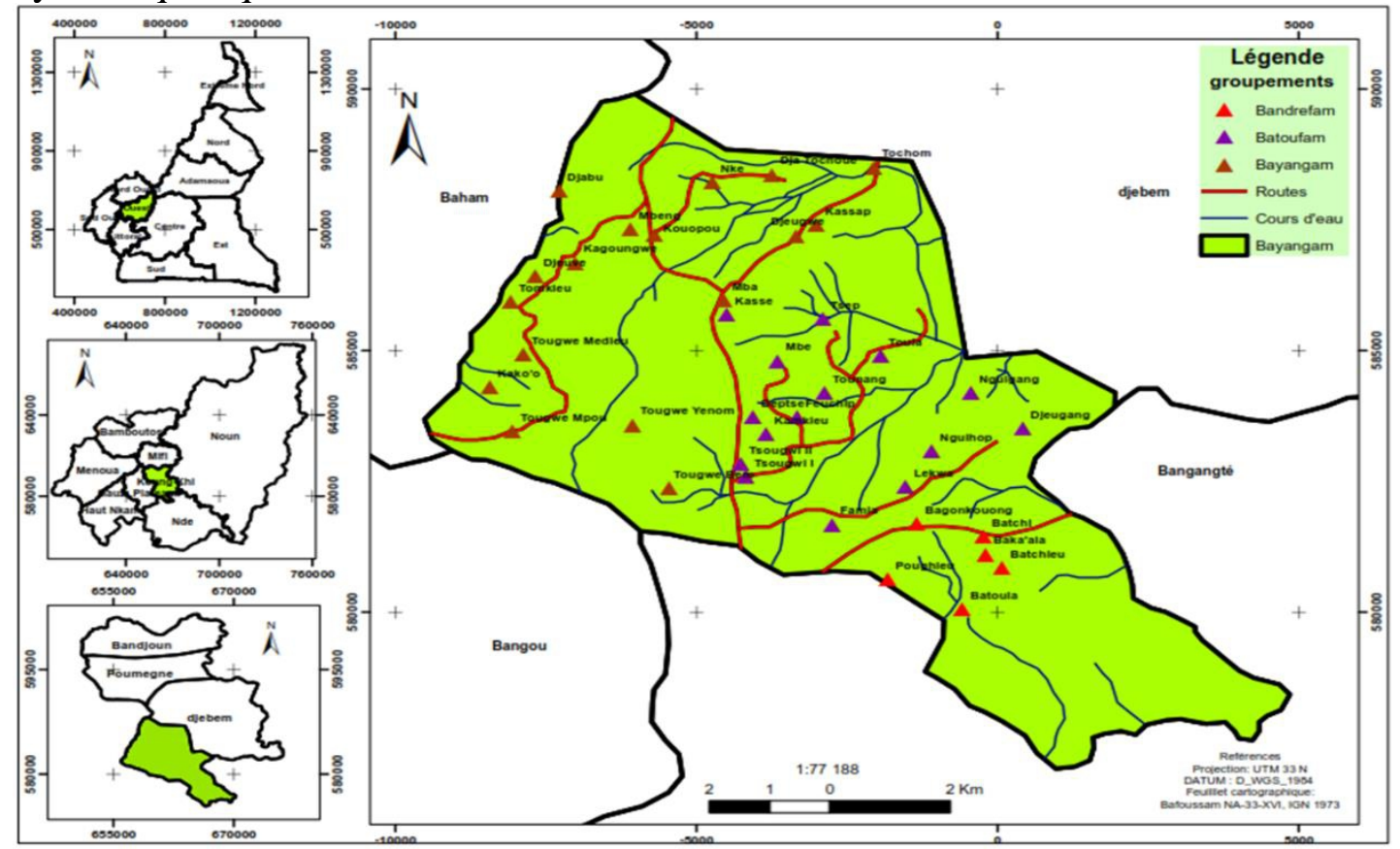

Figure 1 : Carte de localisation de la zone d'étude.

Ces acteurs, à travers la création des Comités Villageois de Gestion des Points d'Eau (CVGPE) oeuvrent pour le bon fonctionnement des ouvrages parfois collectivement construits. Malgré la mise en place de ces CVGPE, force est de constater que de nombreux ouvrages sont en mauvais état, et de ce fait ne garantissent pas l'accès pérenne à la ressource en eau de bonne qualité dans cette localité. De ce constat, est née une question essentielle à la base de cette recherche à savoir : comment l'existence des CVGPE n'assure pas de manière pérenne et durable la gestion des points d'eau et par conséquent

${ }^{1}$ L'arrondissement de Bayangam est situé entre $5^{\circ} 14^{\prime}$ et $5^{\circ} 19^{\prime}$ de latitude Nord et de $10^{\circ} 14^{\prime}$ et $10^{\circ} 23,8^{\prime}$ de longitude Est. Sa superficie est estimée à $115 \mathrm{~km}^{2}$. Il est limité au nord par l'arrondissement de Poumougne, au sud et à l'est par l'arrondissement de Bangangté et à l'ouest par l'arrondissement de Bangou. 
l'accessibilité des populations de l'arrondissement de Bayangam à l'eau potable?

Pour répondre à cette question, une méthodologie à trois niveaux a été mise en œuvre. D'abord, l'observation directe dans la zone d'étude, puis l'exploitation de la littérature, enfin la collecte des données primaires auprès des acteurs de la gestion des points d'eau dans l'arrondissement de Bayangam. Dans le cadre de la collecte des données primaires, les enquêtes à l'aide d'un questionnaire se sont intéressées à cent-cinquante (150) ménages répartis dans les trois (03) groupements que compte l'arrondissement de Bayangam (à raison de cinquante-cinq (55) pour la groupement Bayangam, soixante-cinq (65) et trente (30) respectivement pour le groupement Batoufam et le groupement Bandrefam). En suite, les entretiens semi-structurés ont été menés auprès de quatorze (14) personnes ressources, notamment les personnes issues de la commune de Bayangam, les chefs de groupement, les élites et les bailleurs de fonds engagés dans la mise en place et la gestion des points d'eau dans l'arrondissement de Bayangam. Enfin, les données spatiales, utiles à la cartographie des points d'eaux ont été collectées à l'aide d'un GPS su l'ensemble du groupement Bandrefam.

Le traitement des données a permis tour à tour: d'analyser l'organisation et le mode de fonctionnement des différents CVGPE, la situation actuelle des points d'eaux de Bayangam et les mécanismes de gestion. Un regard a également été porté sur le jeu d'acteurs, les systèmes de distributions et les contraintes rencontrées. Au terme de ces analyses, quelques pistes de solutions sont formulées pour une meilleure gestion des points d'eaux en zone rurale.

\section{Les Comités Villageois de Gestion des Points d'Eau : mise en place, organisation et mode de fonctionnement}

\subsection{Mise en place et spatialisation des Comités Villageois de Gestion des Points d'Eau}

Sous l'égide de la Commune de Bayangam, l'arrondissement du même nom a vu se mettre en place depuis 1998, dix-huit (18) CVGPE (figure 2). Ces CVGPE sont le résultat des regroupements des populations par quartier où sont implantés les points d'eau. Ces populations à l'issue de ce regroupement désignent les membres du CVGPE par voie d'élection. 


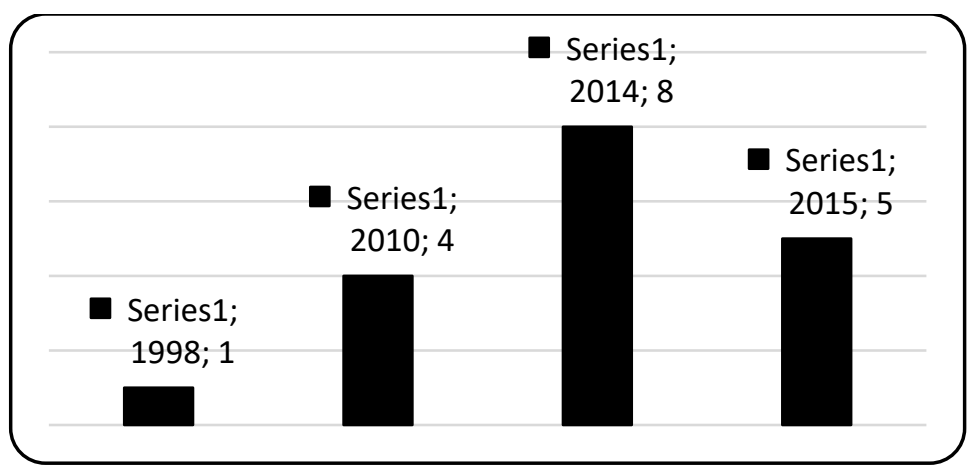

Figure 1: Année de création des CVGPE dans la commune de Bayangam.

Depuis 1998, l'on constate une mise en place progressive des CVGPE dans la Commune de Bayangam soit une (01) en 1998, quatre (04) en 2010 et huit (08) en 2014. Cette situation laisse croire que la majeure partie des comités a été créée au cours des deux dernières décennies. Ceci traduit ainsi, la volonté récente des autorités communales de Bayangam à mieux s'occuper du service de l'eau dans la localité.

Ces CVGPE, en fonction du nombre d'ouvrages qu'elles comportent, sont répartis dans les groupements Bayangam, Bandrefam et Batoufam (figure 2), avec une forte concentration des CVGPE dans le groupement Batoufam (soit 10 CVGPE), contrairement aux groupements Bayangam et Bandrefam qui ont chacun quatre (04) CVGPE. Ceci est dû sans doute au fait que Batoufam est le groupement le plus important des groupements que compte l'arrondissement en terme de superficie, de population et de concentration des points d'eau.

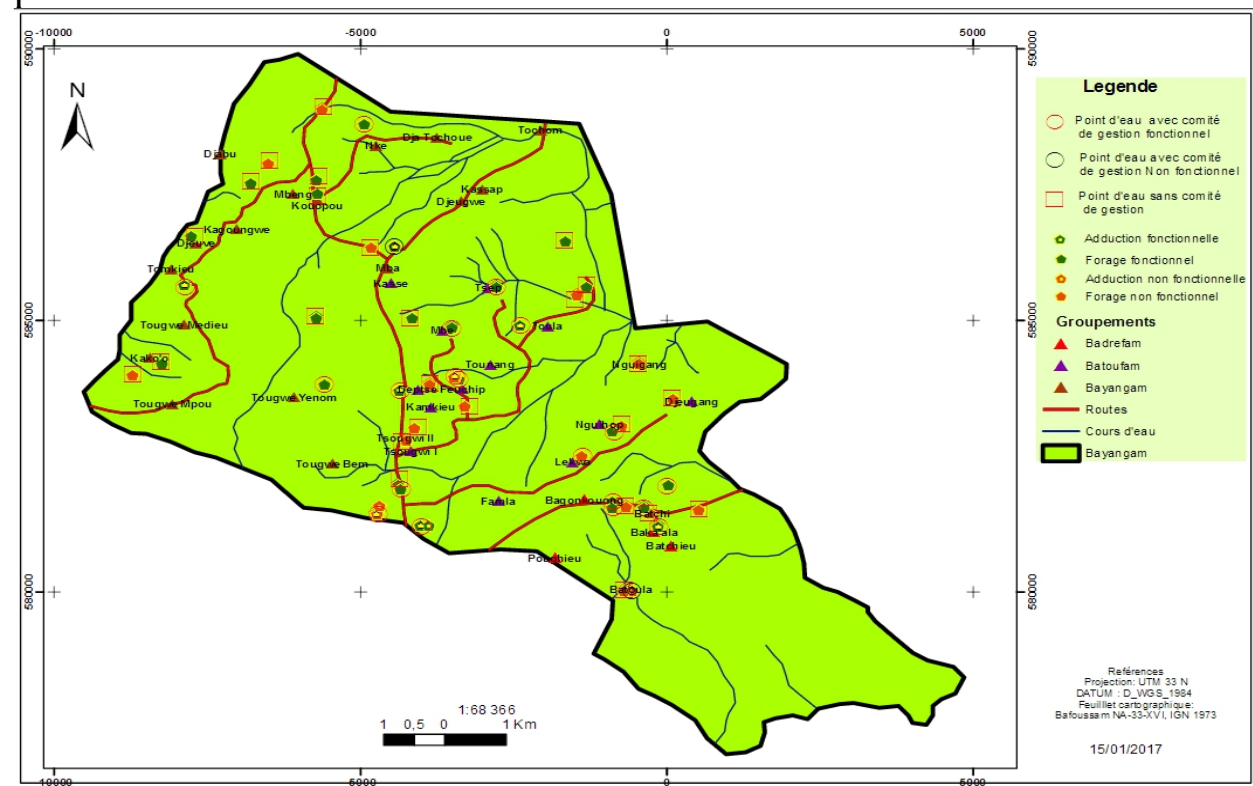

Figure 2 : Répartition spatiale des Comités Villageois de gestion des Points d'Eau à Bayangam 
Malgré cette dynamique de création des CVGPE, un nombre important de point d'eau demeurent sans CVGPE. On note que $43 \%$ de points d'eau n'ont pas toujours de CVGPE. Aussi, parmi les 57\% de points d'eau ayant chacun un CVGPE, il est important de relever que près de $20 \%$ de ces CVGPE sont non fonctionnel.

\subsection{Organisation et fonctionnement des Comités Villageois de Gestion des Points d'Eau}

Les CVGPE ont la charge d'organiser les réunions pour discuter des problèmes ou sujets relatifs à l'état de fonctionnement des points d'eau, mais aussi pour résoudre tous les problèmes ayant trait à la gestion des points d'eau, promouvoir l'utilisation hygiénique et effective des différents services qu'offre l'eau potable, éduquer la communauté et lui rendre de bons services, de s'enquérir auprès d'autres organisations des méthodes de réhabilitation des ouvrages hydrauliques si nécessaire, de contrôler les activités du fontainier et organiser des cérémonies de collecte de fonds au nom de la communauté et rendre régulièrement compte de ses activités à ladite communauté. En réalité, les CVGPE sont des groupes de personnes bénévoles composés d'un président, d'un vice-président, d'un secrétaire, d'un vice-secrétaire, d'un trésorier, d'un commissaire au compte, d'un animateur sanitaire, d'un artisan réparateur, d'un fontainier et dans certains cas d'un ou plusieurs conseillers. Ces membres ont chacun un rôle bien défini et consigné dans le règlement intérieur. Ce dernier étant essentiellement un code de conduite qui régit le fonctionnement du comité. Comme on peut le constater, les CVGPE sont composés des manageurs mais aussi du personnel technique en charge de la réparation des points d'eau en cas de panne. Cependant, $38 \%$ de ces membres n'ont jamais suivi de formation en rapport avec la gestion de l'eau en générale, contre $62 \%$ des membres des comités ont été formés. Avec un nombre aussi important de membres non formés, on peut s'attendre à un difficile fonctionnement de certains CVGPE. En effet jusqu'à 20\% de CVGPE de Bayangam ne fonctionnent presque pas. Les structures non fonctionnelles étant le résultat de l'inexpérience de certains membres et de surcroît non formés, mais également des activités liées aux mécanismes de gestion des points d'eau. Ainsi et comme le fait remarquer Olivier De Sardan (2000) à partir du cas de la gestion des points d'eau dans le secteur de l'hydraulique villageoise au Niger et en Guinée, les comités de gestion apparaissent comme les points faibles de la gestion de l'eau en milieu rural. 


\section{Cartographie, mécanismes de gestion et état de fonctionnalité des points d'eau}

A Bayangam, les points d'eaux sont soumis à des mécanismes de gestion appropriés. Ces différents mécanismes déterminent ainsi l'état de leur fonctionnement.

\subsection{Répartition spatiale des différents points d'eau à Bayangam}

Les points d'eau que l'on retrouve dans l'arrondissement de Bayangam sont pour la plus part, des forages et adductions d'eau. Ceux-ci sont tantôt fonctionnels, tantôt non fonctionnels. L'arrondissement de Bayangam en compte précisément quarante-six (46). Ces derniers sont malheureusement inégalement répartis sur l'ensemble des trois groupements (tableau 1). En effet, le groupement Batoufam compte à lui seul vingt-deux (22) points d'eau, contre treize (13) et onze (11) point d'eau respectivement pour les groupements Bayangam et Bandrefam. Un fait important qu'il faut souligner ici, est que de nombreux villages du groupement Bayangam, ne disposent pas d'un grand nombre de points d'eau pourtant ce groupement est celui qui regroupe le plus grand nombre de village de l'arrondissement. Les points d'eau de ses différents groupements sont en effet les forages (38), et les adductions d'eau composés essentiellement de bornes fontaines (08).

Tableau 1 : Distribution spatiale des points d'eau dans l'arrondissement de Bayangam

\begin{tabular}{|c|c|c|c|c|c|c|}
\hline Groupements & \multicolumn{2}{|l|}{ Bayangam } & \multicolumn{2}{|l|}{ Batoufam } & \multicolumn{2}{|l|}{ Bandrefam } \\
\hline \multirow{18}{*}{$\begin{array}{l}\text { Nombre } \quad \text { de } \\
\text { forages } \\
\text { village }\end{array}$} & Kouopou & 02 & Leukwa & 03 & Bakaala & 02 \\
\hline & Kako’o & 02 & Tsougwi 2 & 03 & Bagonkouong & 05 \\
\hline & Yenom & 01 & Tsep & 02 & Poughieu & 02 \\
\hline & Nké & 01 & Feuchip & 02 & Batchi & 01 \\
\hline & Tomkieu & 01 & Toula & 03 & Batchieu & 01 \\
\hline & Djabu & 01 & Nguigang & 01 & - & - \\
\hline & Djeuve & 01 & Djeugang & 01 & - & - \\
\hline & Mba & 02 & Kasse & 01 & - & - \\
\hline & $\begin{array}{l}\text { Tougwe- } \\
\text { Meudjeu }\end{array}$ & 01 & Kamkieu & 01 & - & - \\
\hline & $\begin{array}{l}\text { Djeugwe- } \\
\text { Kassap }\end{array}$ & 01 & Depste & 02 & - & - \\
\hline & Dja-tochoue & - & Tsougwi 1 & 02 & - & - \\
\hline & Mbeng & - & Tounang & 01 & - & - \\
\hline & Kassap & - & Mbe & - & - & - \\
\hline & Tochom & - & Nguihop & - & - & - \\
\hline & Djeugwe & - & - & - & - & - \\
\hline & Tougwe-Bem & - & - & - & - & - \\
\hline & Tougwe-Mpou & - & - & - & - & - \\
\hline & Kagongwe & - & - & - & - & - \\
\hline Total & 13 & & 22 & & 11 & \\
\hline
\end{tabular}




\subsection{Mécanismes de gestion des points d'eau}

L'exploitation des points d'eau intègre d'une part leur gestion sanitaire, qui consiste à amener les usagers à mettre en œuvre les mesures d'hygiènes autour des points d'eau. D'autre part, elle nécessite la maintenance des points d'eau qui suppose la pratique d'un ensemble d'opérations d'entretien technique, ceci dans le souci d'accroître sa fiabilité, sa disponibilité et sa durée de vie. En outre, il faut souligner que la bonne gestion des points d'eau de Bayangam nécessite également le fonctionnement et la sécurité des ouvrages, et enfin la gestion financière des points d'eau (gestion des caisses ou comptes bancaires qui existent au sein des CVGPE). Si les autres types de gestion sont importants pour la durabilité des points d'eau, la gestion financière est le point le plus important, car une bonne gestion financière entraine l'amélioration des autres types de gestions. En effet, la collecte et l'utilisation harmonieuse des ressources financières sont à la base de la gestion sanitaire et de la maintenance des points d'eau. Or dans l'arrondissement de Bayangam, 53,85\% de CVGPE ne disposent ni de caisse, ni de compte bancaire pour sécuriser la mobilisation et l'utilisation des ressources financières, ceci parce que les populations ne contribuent pas, ou encore parce que l'argent collecté est confiné entre les mains d'un seul membre du bureau sans document de comptabilité. Pourtant, les contributions faites par les différentes parties prenantes sont prioritairement destinées aux charges relatives à l'entretien, la maintenance, le fonctionnement des forages, l'exploitation et l'extension du réseau d'adduction d'eau. Selon les cahiers de charge, la commune ne prend en charge les dépenses de fonctionnement que si elles excèdent la somme de cent mille francs.

Par ailleurs, il est important de remarquer que la participation financière des populations bénéficiaires s'inscrit dans une dynamique d'appropriation de l'ouvrage hydraulique. En l'absence de mobilisation financière, la plupart d'acteurs ne se sentent pas très concernées par les activités de développement comme le faisait remarquer Goehrs (2017). Ce qui ne contribue pas à assurer un meilleur fonctionnement des points d'eau.

\section{3. État de fonctionnement des points d'eau}

La construction des points d'eau dans l'arrondissement de Bayangam n'a pas permis de résoudre le problème d'accès à l'eau potable des populations. Il existe en effet un fort contraste entre la création des points d'eau et leur fonctionnement. La plupart des points d'eau ne joue pas le rôle à eux assigné du fait de leur non fonctionnement car sur huit (08) adductions, six (06) sont fonctionnels tandis que deux (02) sont non fonctionnels. Sur trente-huit (38) forages, vingt (20) sont fonctionnels et dix-huit (18) ne le sont pas. Ce qui revient à un total de vingt-six (26) points d'eau fonctionnels contre vingt (20) non fonctionnels soit $43,47 \%$ de structures non fonctionnel à 
Bayangam. Par ailleurs, si le taux de non fonctionnement est relativement faible, $33,33 \%$, il est très élevé dans la catégorie des forages avec 90 d'infrastructures non fonctionnels (tableau 2), sans doute peu adaptées ou difficile d'entretien.

Tableau 2 : Etat de fonctionnement des points d'eau dans l'arrondissement de Bayangam

\begin{tabular}{|l|l|l|l|l|}
\hline \multirow{2}{*}{$\begin{array}{l}\text { Type de points } \\
\text { d'eau }\end{array}$} & \multicolumn{2}{|l|}{ Etat de fonctionnement } & $\begin{array}{l}\% \text { de non } \\
\text { fonctionnement par } \\
\text { type d'ouvrage }\end{array}$ \\
\cline { 2 - 6 } & Fonctionnel & Non fonctionnel & Total & - \\
\hline Adduction & 06 & 02 & 08 & 33,33 \\
\hline Forage & 20 & 18 & 38 & 90 \\
\hline Total & 26 & 20 & 46 & 43,47 \\
\hline
\end{tabular}

Les photos 1 et 2 présentent deux ouvrages hydrauliques (un forage et une borne fontaine) non fonctionnels respectivement dans les villages Tsougwi 2 et Kassap.

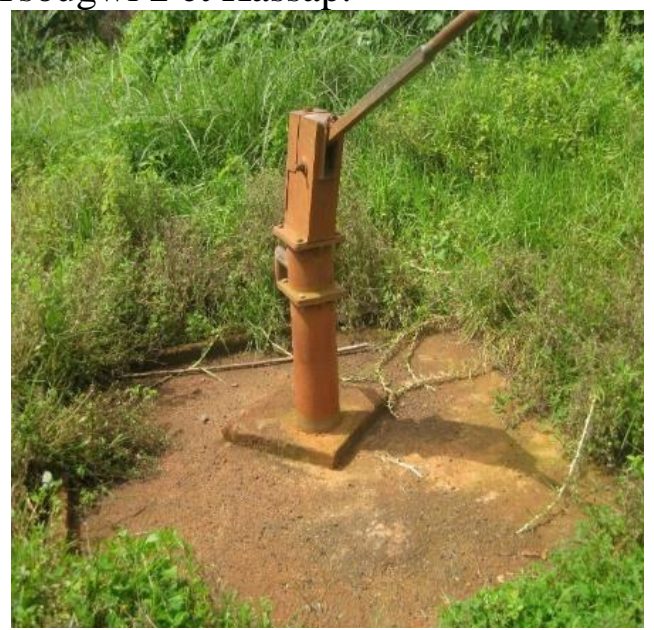

Cliché : Noufin, 2016.

Photo1 : Forage en panne et abandonné au village Tsougwi 2

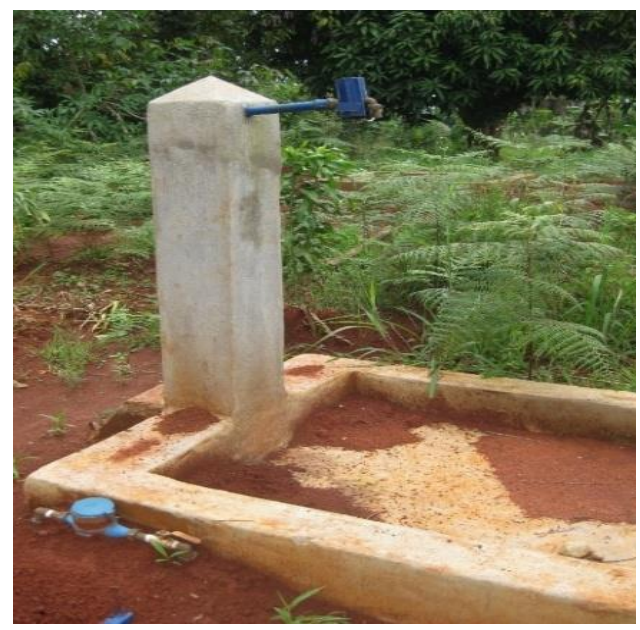

Cliché : Noufin, 2016.

Photo 2 : Borne fontaine en panne à Kassap

Par ailleurs, les points d'eau fonctionnels quant à eux sont en très mauvais état de salubrité. Les photos 3 et 4 , présentent d'une part un forage mal entretenu, pourtant fonctionnel et utilisé à plein temps et d'autre part un château d'eau progressivement envahi par la végétation. Pourtant, il s'agit bien des ouvrages en cours d'utilisation en cours. 


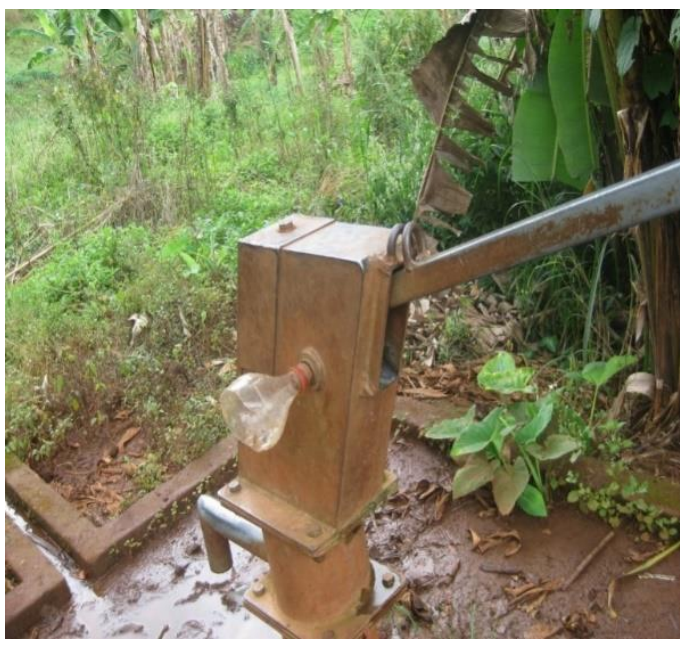

Cliché Noufin (2016)

Photo 3: Forage fonctionnel à Tsougwi 1

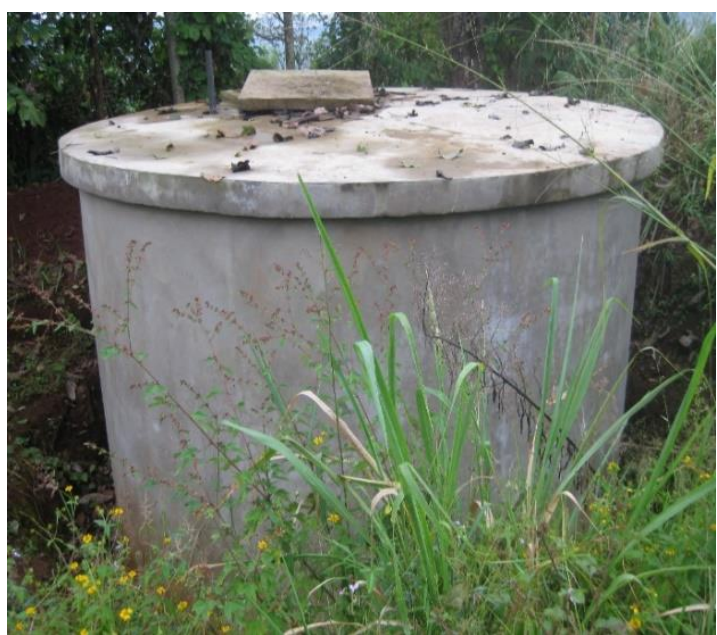

Cliché Noufin, 2016

Photo 4: Château d'eau de l'adduction de Tounang-Toula

Il est important de remarquer que la situation des points d'eau de Bayangam est le reflet de la plupart des projets d'adduction dans certains du Cameroun. C'est le cas par exemple des point d'eau à Maroua dans le Nord Cameroun qui présentent un état de non fonctionnement entre 22 et $44 \%$ selon les locallités (Hassana, 2010). Si les questions de gestion financière sont à la base du mauvais ou non fonctionnement des points d'eau, il faut remarquer que le manque d'appropriation par les utilisateurs mais aussi le jeu d'acteurs justifient la situation des points d'eau dans l'arrondissement de Bayangam.

\section{Acteurs, financement des points d'eau et accessibilité à l'eau par les populations}

\subsection{Acteur et financement de la construction des points d'eau}

De nombreux acteurs interviennent dans la gestion des points d'eau dans l'arrondissement de Bayangam. En plus des CVGPE, chargés de la gestion quotidienne des points d'eau, les acteurs qui interviennent dans le financement de la mise en place des points d'eau dans l'arrondissement Bayangam sont nombreux. Par ordre d'importance, on distingue 1'Etat Camerounais à travers le Budget d'Investissement Public (BIP), avec 69,56\% de points d'eau financés, les particuliers, avec 10,86\% de points d'eau financés, la Mairie de Bayangam, avec 6,5\% de points d'eau financés, la Banque Africaine de Développement et l'ONG Lumière du Monde, avec chacune $4,34 \%$ de points d'eau financés, et le Don Chinois et Initiative d'Allègement de la Dette Multilatérale (IADM), avec chacun 2,1\% de point d'eau de point d'eau financés (tableau 3). 
Tableau 3 : Acteurs de financement de la construction des points d'eau

\begin{tabular}{|l|l|l|}
\hline Source de financement & Fréquence & \% \\
\hline Banque Africaine de Développement (BAD) & 2 & 4,34 \\
\hline État (Budget d'Investissement Public) & 32 & 69,56 \\
\hline Don Chinois & 1 & 2,1 \\
\hline Initiative d'Allègement de la Dette Multilatérale (IADM) & 1 & 2,1 \\
\hline Mairie de Bayangam & 3 & 6,5 \\
\hline ONG Lumière du monde & 2 & 4,34 \\
\hline Particuliers (élites) & 5 & 10,86 \\
\hline Total & $\mathbf{4 6}$ & $\mathbf{1 0 0}$ \\
\hline
\end{tabular}

En dehors de la Mairie de Bayangam, tous les autres acteurs dans le financement et la mise en place des points d'eau sont extérieurs au territoire et ne participent pas à la gestion quotidienne des ouvrages. Ils se limitent à la mise en place, laissant la gestion aux mains des CVGPE déjà peu scrupuleux pour la plupart, mal formés ou pas du tout formé. Aussi, au sein des CVGPE, il se joue des jeux de positionnement et de leadership entre les membres et dont le seul objectif est la quête du pouvoir et non la gestion de l'institution et des points d'eau. On a relevé pendant les enquêtes la présence de branchements frauduleux (soit 08) sur les réseaux d'adduction d'eau. Ceci est malheureusement fait avec la complicité de certains membres des CVGPE.

\subsection{Les mécanismes d'accessibilité à l'eau par les populations}

Dans le cadre de l'accès des populations à l'eau, le service de puisage est assuré par un fontainier ou une fontainière ayant au mieux la position la plus proche du point d'eau, mais aussi le plus disponible. Ce dernier a la charge d'ouvrir et de fermer le forage ou la borne fontaine, et dans certains cas la vanne à des heures déterminées. Bien des fois, l'on s'est rendu compte malheureusement que cet acteur, n'assume pas toujours son rôle de façon efficiente.

L'accès à l'eau par ailleurs, est soumis dans la plupart de cas au payement des frais fixés par chaque comité. Ces taux varient de 500 FCFA par mois à 2500 FCFA pour des usages ponctuels. Pour certains points d'eau comme celui de Djeugwe-Kassap ou encore de Tounang-Toula, des mécanismes de comptage ont été mis en place et les frais s'élèvent à quarantemilles francs $(40000 \mathrm{Fcfa})$ car le mètre cube varie entre $150 \mathrm{FCFA}$ et 200 FCFA. Cet accès monétarisé à l'eau pourtant nécessaire pour la bonne gestion des points d'eau, exclut cependant un nombre important de ménages qui préfèrent continuer à s'approvisionner dans les rivières et marigots au lieu de payer au CVGPE la somme demandée. C'est ce qui justifie sans doute le fait que les caisses de certains CVGPE soient relativement vides, avec un impact 
significatif sur le renouvellement des ouvrages ou leur entretien en cas de panne.

\section{Vers un système de gestion durable des points d'eau en zone rurale}

La gestion durable des points d'eau est inéluctablement en relation avec l'intervention des acteurs. Elle dépend des recommandations faites principalement au niveau de la commune, des CVGPE et des populations qui sont des acteurs clés de la gestion des points d'eau.

\subsection{Au niveau de la commune}

Le diagnostic complet de tous les points d'eau de Bayangam est recommandé à la commune de Bayangam pour une meilleure réhabilitation des ouvrages défectueux de même que la construction des points d'eau de qualité durable, qui doit tenir compte des zones déficitaires. Un appui considérable aux comités de gestion est également envisageable, ceci à travers la création d'un budget communal qui doit être approvisionné par les différents comités et devant servir pour prévenir les cas de pannes graves.

\subsection{Au niveau des CVGPE}

L'intérêt général doit primer sur les intérêts individuels. Les membres doivent être davantage disponibles, surtout les techniciens en charge du dépannage des ouvrages. Aussi, la collaboration avec les populations bénéficiaires doit être améliorée ainsi que l'organisation des assemblées générales au cours desquelles des comptes rendus doivent être faits aux populations bénéficiaires.

\subsection{Au niveau des populations}

Pour que les activités relatives à l'entretien des points soient davantage efficaces, les populations bénéficiaires sont appelées à mieux s'investir notamment à travers le respect des règles d'hygiène et de salubrité autour des points d'eau, la mobilisation des contribution financière pour appuyer les CVGPE et le respect des modalités de consommation de la ressource en eau fixées par les différents CVGPE.

\section{Conclusion}

$\mathrm{Au}$ terme cette recherche au cours duquel les CVGPE et les points d'eau dans l'arrondissement de Bayangam ont été interrogés, nous notons que les populations dudit arrondissement sont dans un état de vulnérabilité certaine suite au difficile accès à l'eau en quantité et en qualité. En effet, les comités en charge de la gestion de ces ouvrages en plus d'être pour certains non fonctionnels, ne rendent pas un service de qualité plus ou moins satisfaisant à 
cause d'une mauvaise organisation et d'un système de fonctionnement non adéquat et inefficace. Par ailleurs, ces institutions ne sont pas suffisamment soutenues par la commune de Bayangam qui a pourtant dans la plupart de cas contribué à les mettre en place. Les usagers, de par leur comportement et leur manque d'engagement ne favorisent pas le bon déroulement des travaux relatifs à la gestion des points d'eau. C'est fort de cet état de choses que quelques solutions ont été proposées afin de contribuer à une meilleure gestion des points d'eau dans l'arrondissement de Bayangam en particulier et en zone rurale en générale. Ces propositions sont essentiellement relatives aux principaux acteurs qui interviennent dans la gestion des points d'eau, notamment la commune de Bayangam, les CVGPE et les populations. Ces acteurs doivent être davantage responsables pour une gestion plus durable des points d'eau.

\section{References:}

1. Barron, C. et Tidjani, A. (2011). L'accès à l'eau en Afrique subsaharienne : au-delà des modèles, une pluralité d'innovations locales, Mondes en développement, $\mathrm{n}^{\circ} 155,7-22$.

2. Goehrs, M. (2017). Budgets participatifs : s'approprier la dialectique de la gouvernance participative locale, L'Année du Maghreb, 16, 223244.

3. Hassana, (2010). Gestion des forages d'eau et diagnostic-solution Dans la communauté urbaine de Maroua (Cameroun), mémoire pour l'obtention du Master Spécialisé en Génie Sanitaire et Environnement (GSE), Institut International d'Ingénierie de l'Eau et de l'Environnement (2iE), 57p

4. Helvetas Cameroon (2003). Manuel de formation de Comités Villageois de gestion d'eaux villageoise, Helvetas Cameroon, 51p.

5. Ndongo, B., Ngnikam, E et Lako Mbouendeu, S. (2012), Analyse des services d'eau non conventionnels dans les quartiers à habitat spontané : cas du quartier Eba'a dans la périphérie de la ville de Yaoundé (région du centre au Cameroun), Erudit vol 25, numéro 2, Ed. Université du Québec-INRS-Eau, Terre et Environnement (INRS-ETE), 12p.

6. Olivier De Sardan J-P. (dir). (2000). La gestion des points d'eau dans le secteur de l'hydraulique villageoise au Niger et en Guinée, AFD, Rapport d'Etude, http://hubrural.org/IMG/pdf/afd_gestion_des_points_deau.pdf, consulté en ligne le 30 Août 2018.

7. Schneier-Madanes, G. (ed.). (2011). L'eau mondialisée, la gouvernance en question. Paris, La Découverte.490p. 
8. Tayeb, Kasmi, M. (2007), Manuel des analyses socioéconomiques et de gestion des points d'eaux, vol 2: Gestion des points d'eaux, Bischofliches Hilfswerk Misereor e. v., 96p. 